\title{
Glial Chain Migration Requires Pioneer Cells
}

\author{
Benoît Aigouy, Léa Lepelletier, and Angela Giangrande \\ Institut de Génétique et Biologie Moléculaire et Cellulaire, Centre National de la Recherche Scientifique, Université Louis Pasteur, Institut National de la \\ Santé et de la Recherche Médicale, 67404 Illkirch, Communauté Urbaine de Strasbourg, France
}

The migration of glial chains along the nerve entails directional and coordinated movement. Despite its importance in the formation of the nervous system, this process remains poorly understood, because of the difficulty of manipulating identified cells. Using confocal time-lapse and cell ablation in the whole animal, we provide direct evidence for a discrete number of Drosophila peripheral glial cells acting as pioneers and guiding the rest of the migratory chain. These cells are in direct contact with several follower cells through a very long and stable cytoplasmic extension. The presence of pioneer cells and homotypic interactions at the tip of the chain allows coordinated movement and the formation of a continuous sheath around the nerve. These in vivo data open novel perspectives for understanding the cellular bases of vertebrate glial migration in physiological and pathological conditions.

Key words: glia; Drosophila; migration; pioneers; chain; time-lapse

\section{Introduction}

Glia are known to move extensively to reach their final destination, often migrating as groups of cells (Sepp et al., 2000; Van De Bor et al., 2000; Lemke, 2001; Gilmour et al., 2002; Tsai and Miller, 2002; Silies et al., 2007; von Hilchen et al., 2008). Collective migration involves not well understood pathways of cell communication allowing sheets, strands, clusters and chains to move coordinately as a functional unit. The movement of large number of cells stretching considerable distance, typical of migratory chains, calls for strong driving forces being at work. In addition, the presence of highly mobile cells at the leading edge suggests that a specialized population of cells guides the rest of the chain (for review, see Lecaudey and Gilmour, 2006). The difficulty of manipulating individual glial cells and nervous tissue complexity, however, make it hard to test this hypothesis at the single cell level.

Because of their simple organization, peripheral Drosophila glia constitute an ideal system to analyze the cellular cues regulating collective migration (Giangrande, 1994; Sepp et al., 2000; Aigouy et al., 2004; Silies et al., 2007; von Hilchen et al., 2008).

\footnotetext{
Received Feb. 13, 2008; revised July 20, 2008; accepted Sept. 20, 2008.

This work was supported by the European Economic Community (Grant QLG3-CT-2000-01224), Institut National de la Santé et de la Recherche Médicale, Centre National de la Recherche Scientifique, the Hôpital Universitaire de Strasbourg, Ministère de la Recherche (Action Concertée Incitative Développement), Fondation pour la Recherche Médicale (FRM), Agence Nationale de la Recherche, Ligue Nationale contre le Cancer, and Association pour la Recherche sur le Cancer (ARC). B.A. was supported by Mission de la Recherche et de la Technologie, ARC, and FRM fellowships. We thankS. Leevers, V. Auld, C. Desplan, L. Luo, the Bloomington stock centre, Cancer Research UK, and the Developmental Studies Hybridoma Bank for flies, antibodies, and plasmids. We thank J. L. Vonesch, M. Boeglin, and D. Hentsch for help with confocal microscope, imaging, and data processing. We thank C. Diebold for excellent technical help, S. Berszenyi for sharing unpublished results, S. Bour for preparing figures, group members for helpful comments, and L. Soustelle for manuscript editing.

Correspondence should be addressed to Angela Giangrande, Institut de Génétique et de Biologie Moléculaire et Cellulaire, CNRS/INSERM/Université Louis Pasteur, B.P.10142, 67404 Illkirch Cedex, Communauté Urbaine de Strasbourg, France. E-mail: angela@titus.u-strasbg.fr.

B. Aigouy's present address: Max Planck Institute, Pfotenhauer Strasse 108, 01307 Dresden, Germany.

L. Lepelletier's present address: Institut Pasteur, 75015 Paris, France.

D0I:10.1523/JNEUROSCI.1361-08.2008

Copyright $\odot 2008$ Society for Neuroscience $\quad 0270-6474 / 08 / 2811635-07 \$ 15.00 / 0$
}

Glial cells present in the Drosophila wing migrate over two major sensory nerves, a process that takes several hours and allows uniform nerve ensheathment (Giangrande, 1994; Van De Bor et al., 2000; Aigouy et al., 2004). The glial chain present in the anterior margin or L1 nerve contains more than seventy cells spanning $>500 \mu \mathrm{m}$. Glia follow axons and interact with them during development: glia stop migrating if axons are stalled and they change direction of migration if axons follow novel routes (Giangrande, 1994; Aigouy et al., 2004). The L1 glial chain is populated by morphologically different cells: tip cells send long exploratory filopodia, actin-based extensions that grow and retract rapidly, whereas cells within the chain are spindle shaped and much less dynamic (Aigouy et al., 2004).

By cell ablation, we here show that few glia at the L1 chain tip promote directional migration of the rest of the chain. Their absence results in strong delay or complete arrest of migration. Their role is similar to that of pioneer axons, which allow follower, late, axons to navigate with high fidelity along stereotyped routes. Glial pioneer cells are able to contact several followers by extending a long cytoplasmic process at their rear end. Finally, pioneer glia are required throughout the migratory process and promote chain integrity via retrograde interactions with follower cells. These in vivo data demonstrate that glial chain migration is a highly coordinated process that is based on homeostatic interactions.

\section{Materials And Methods}

Fly strains and heat shock treatments. Flies were raised at $25^{\circ} \mathrm{C}$. repoGal4 (gift from V. Auld, University of British Columbia, Vancouver, British Columbia, Canada) was used to drive expression of the $U A S-n c G F P$ reporter (nc: nuclear and cytoplasmic) (gift from C. Desplan, New York University, New York, NY) and UAS-CD8GFP (membrane) (Lee et al., 2000) (Bloomington stock center) in glia (Repo::ncGFP/Repo::CD8GFP). The DsRed Express (Bevis and Glick, 2002) gene was used to produce elav-DsRed express flies. MARCM was performed in FRT19A/FRT19A, hs-FLP, tub-Gal80; UAS-ncGFP/+; repo-Gal4/+ or FRT19A/FRT19A, hs-FLP, tub-Gal80; UAS- 
CD8GFP/+; repo-Gal4/+ flies. Clones were obtained after a $37^{\circ} \mathrm{C} 1 \mathrm{~h}$ heat-shock at wander-

ing L3.

Immunohistochemistry. Immunolabeling was performed as in (Giangrande et al., 1993) using mouse anti-Repo (1:1000) (DSHB), rat anti-Elav (1:500) (DSHB) and rabbit anti-GFP (1:1000) (Invitrogen). Secondary antibodies coupled with FITC, CY3 or CY5 (Jackson) were used at 1:400. Wings were mounted in Vectashield medium (Vector Laboratories).

Time-lapse, UV laser targeting and image processing. Animals for time-lapse and ablations were prepared as in (Aigouy et al., 2004) and imaged in 4D using a TCS SP2 inverted confocal microscope (Leica) equipped with a Ar laser $(488 \mathrm{~nm})$ to excite GFP, a $543 \mathrm{~nm}$ laser to excite DsRed express and a UV laser for ablations. A heating stage maintained temperature constant $\left(25^{\circ} \mathrm{C} \pm 2^{\circ} \mathrm{C}\right)$. Axons of repo::ncGFP; elavDsRed express wings were submitted to UV irradiation $(350 \mathrm{~nm}, 20 \mathrm{~s}$ pulse at medium intensity) using the same protocol as that used for cell ablations.

Z stack projections, rotations, figure mounting and two color time-lapse movies were obtained using house developed TIMT software and ImageJ. To reduce noise, a median filter $3 \times 3$ was applied on red images stacks before projection. Images were annotated using Adobe Photoshop and Adobe Illustrator; movies were converted to the QuickTime format using Adobe Premiere.

\section{Results}

Glial chain migration in the fly wing

Fly wing displays a glial migratory chain along the so-called L1 nerve (Fig. $1 A, B$ ) (Giangrande, 1994; Aigouy et al., 2004). Migration proceeds in a coordinated manner until L1-glia reach more proximal cells called radius-glia, thereby forming a continuous sheath along the nerve. The presence of highly motile cells at the chain tip (Aigouy et al., 2004) and the fact that such cells stay at that position throughout migration (B. Aigouy, unpublished observation), led us to ask whether a specialized glial population triggers and drives migration. We therefore performed ablations in repo::ncGFP animals by confocal mediated UV targeting (Aigouy et al., 2004) and determined whether removing cells at L1 chain tip affects migration. Achievement of L1-radius glial connection was followed by time-lapse. Animals of the same genotype but not submitted to ablation were run in parallel as controls.

Time-lapse on controls shows that L1-glia start migrating between 16 and $18 \mathrm{~h}$ after puparium formation (h APF). In all animals $(n=19)$, migration is achieved between 24 and $27 \mathrm{~h}$ $\mathrm{APF}$, and in fact, almost two thirds of the wings (12 of 19) accomplish migration by $25 \mathrm{~h} \mathrm{APF}$, that is in $1 \mathrm{~h}$ interval from the earliest arrivals (Figs. $1 C, D, 2 A-D$; Movie 1, available at www.jneurosci.org as supplemental material). This constitutes a very robust behavior, considering that the white pupa stage ( $0 \mathrm{~h} \mathrm{APF})$, at which we take animals for subsequent analyses, last on its own $30 \mathrm{~min}$. To gain quantitative informa-

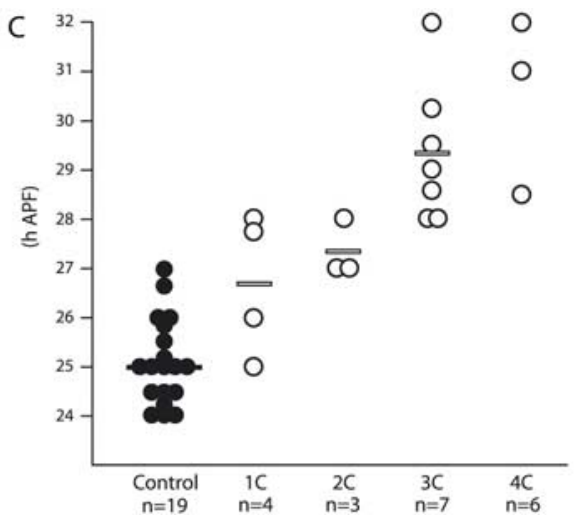

$\begin{array}{lcccc}\text { Control } & 1 C & 2 C & 3 C & 4 C \\ n=19 & n=4 & n=3 & n=7\end{array}$

\begin{tabular}{|l|c|c|c|c|}
\hline \multicolumn{1}{|c|}{ Sets of cell ablations } & $\begin{array}{l}\text { Successful } \\
\text { migration* }\end{array}$ & MI & $\begin{array}{c}\text { Earliest } \\
\text { arrivals }\end{array}$ & Latest arrivals \\
\hline & & & & \\
\hline Control $(\mathrm{n}=19)$ & $100 \%$ & $25 \mathrm{~h} \mathrm{APF}$ & $24 \mathrm{~h} \mathrm{APF}$ & $27 \mathrm{~h} \mathrm{APF}$ \\
\hline 1 to 4 cell ablation $(\mathrm{n}=20)$ & $60 \%$ & $28 \mathrm{~h} \mathrm{APF}^{* *}$ & $25 \mathrm{~h} \mathrm{APF}$ & $\begin{array}{c}\text { Later than } 32 \mathrm{~h} \\
\text { APF }\end{array}$ \\
\hline 1 cell ablation $(\mathrm{n}=4)$ & $100 \%$ & $26 \mathrm{~h} 41 \mathrm{~m}$ APF & $25 \mathrm{~h} \mathrm{APF}$ & $28 \mathrm{~h} \mathrm{APF}$ \\
\hline 2 cell ablation $(\mathrm{n}=3)$ & $100 \%$ & $27 \mathrm{~h} 20 \mathrm{~m} \mathrm{APF}$ & $27 \mathrm{~h} \mathrm{APF}$ & $28 \mathrm{~h} \mathrm{APF}$ \\
\hline 3 cell ablation $(\mathrm{n}=7)$ & $29 \%$ & $29 \mathrm{~h} 20 \mathrm{~m} \mathrm{APF}$ & $28 \mathrm{~h} \mathrm{APF}$ & $32 \mathrm{~h} \mathrm{APF}$ \\
\hline 4 cell ablation $(\mathrm{n}=6)$ & $0 \%$ & Later than $31 \mathrm{~h}$ & $28 \mathrm{~h} 30 \mathrm{~m} \mathrm{APF}$ & $\begin{array}{c}\text { Later than } 32 \mathrm{~h} \\
\text { APF }\end{array}$ \\
\hline
\end{tabular}

Figure 1. Axonal navigation and glial migration in the fly pupal wing. $A, B$, Schematic representation of wings before L1-glia migration (16 h APF) $(\boldsymbol{A})$ and after migration completion (29 h APF) $(\boldsymbol{B})$; glia in green, neurons and nerves in red. Arrow in $\boldsymbol{A}$ indicates direction of migration from anterior margin to the radius. Box in $\boldsymbol{B}$ shows the region of interest that has been analyzed 列 no Ml was calculated for four-cell ablations, because migration is only accomplished in three of six wings. $\boldsymbol{D}$, Summary of chain tip (he first column indicates the experimental conditions (control and ablated wings), the second the percentage destination by $28 \mathrm{~h} \mathrm{APF}$. Double asterisk, MI for the pooled data (one to four cells) is underestimated, because it does not take into account the three samples of four-cell ablation in which migration is not achieved. Same for Ml of four-cell ablations.

tion, we defined several parameters: the percentage of wings showing normal migration, the earliest and latest times of arrival and the Migratory Index (MI, provided as h APF). The MI population score corresponds to the average stage at which L1-glia reach radius-glia (Fig. 1C,D).

\section{Pioneer cells trigger chain migration}

Tip cells were UV irradiated at the time L1-glia start migrating. Because at this stage the chain tip is populated by a discrete number of closely located cells (Aigouy et al., 2004), we targeted several glia and indeed observed a severe migratory defect (data not shown). We then performed a quantitative analysis $(n=20)$. One-cell ablation already induces a migratory delay: in average, L1-glia reach radius-glia at later stages and show a higher MI (26 h $41 \mathrm{~m} \mathrm{APF,} n=4)$ compared with what found in controls (25 h APF) (Figs. 1C,D, 3). Nevertheless, the delay is relatively mild, suggesting that more than one tip cell may be necessary to drive chain migration. Defects are indeed more severe when two ( $\mathrm{MI}=27 \mathrm{~h} 20 \mathrm{~m} \mathrm{APF,} n=3$ ) or three 

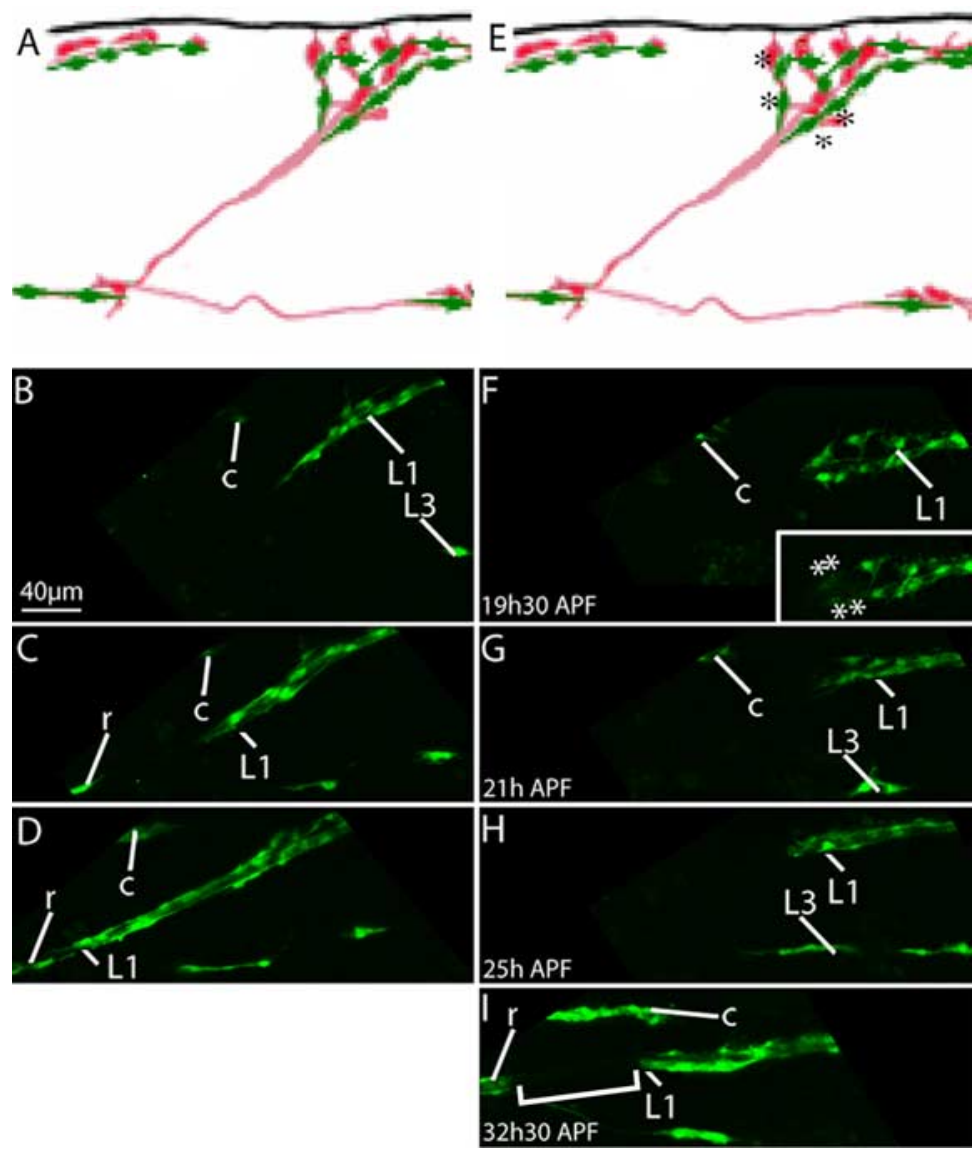

Figure 2. $A-I$, Pioneer cell ablation impairs L1-glia migration. $A, E$, Schematic representations of wing proximal regions at $17 \mathrm{~h}$ APF. Asterisks in $\boldsymbol{E}$ indicate ablated glia; neurons in red, glia in green. $\boldsymbol{B}-\boldsymbol{D}, \boldsymbol{F}-\boldsymbol{I}$, Confocal projections from time-lapse on repo::ncGFP flies: $\boldsymbol{B}-\boldsymbol{D}$, control not submitted to ablation and run in parallel with the wing shown in $\boldsymbol{F}-\boldsymbol{I}$, in which four cells were ablated; indicated stages apply to control and experimental wings. L1, L3, r and c indicate glia on the corresponding nerves. $\boldsymbol{B}-\boldsymbol{D}$, L1-glia have almost reached radius-glia by $25 \mathrm{~h} \mathrm{APF.} \mathrm{F-I,} \mathrm{On} \mathrm{four-cell} \mathrm{ablation,} \mathrm{L1-glia} \mathrm{migration} \mathrm{is} \mathrm{blocked} \mathrm{for} \mathrm{several} \mathrm{hours.} \mathrm{Inset}$ shows L1-glia just after ablation (asterisks indicate targeted glia). The bracket indicates gap between L1-glia and radius glia. Scale bar, $40 \mu \mathrm{m}$.

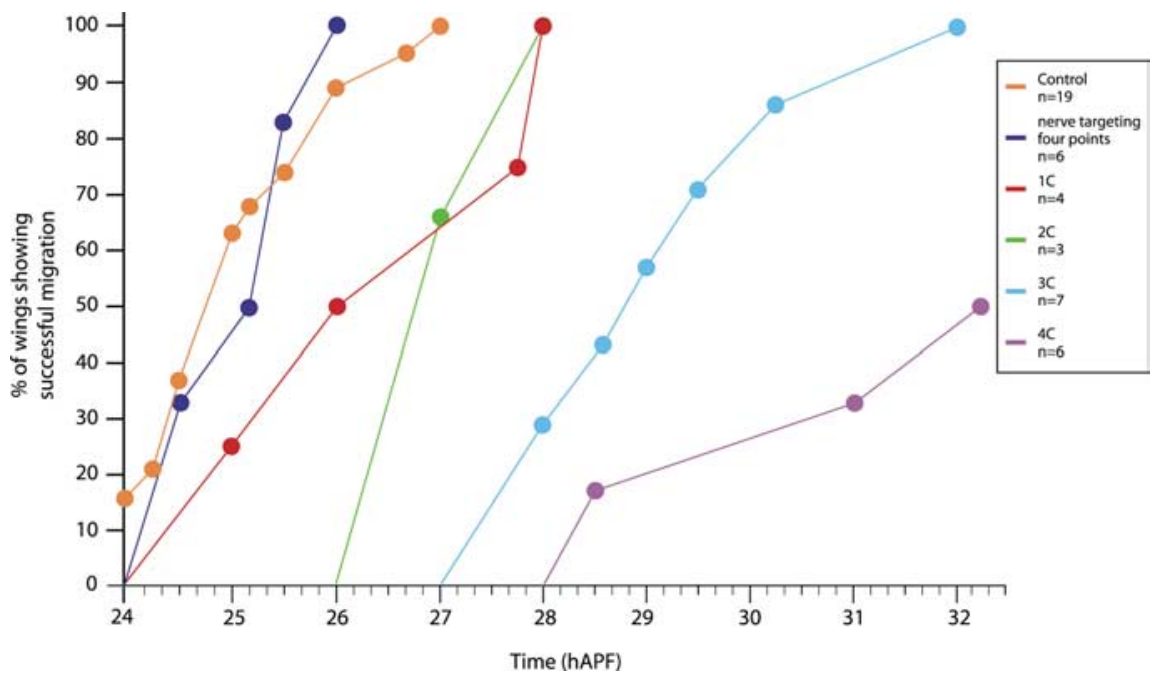

Figure 3. Summary of ablation experiments. The graphic shows the linear progression of migratory delay observed on increasing the number of ablated cells at the chain tip. The $x$-axis, The developmental time in h APF; the $y$-axis, the percentage of wings showing successful L1 glia migration. Experimental conditions as in the text (one to four cell ablation, nerve targeting). In this schematics, samples showing the same arrival time are indicated by a single circle. cells are destroyed (MI $=29 \mathrm{~h} \mathrm{20m} \mathrm{APF,}$ $n=7$ ), in line with the fact that the earliest arrival time is delayed compared with that of controls. Moreover, on three-cell ablation, two thirds of the wings show disconnected glia by the time migration is complete in all controls (Fig. 1C,D).

On four-cell ablation $(n=6)$, none of the samples show complete migration by the time controls show a uniform glial sheath (Figs. $1 C, D, 3$ ). In several cases, L1glia do not move at all for several hours after ablation (Fig. 2E-H; Movie 1, available at www.jneurosci.org as supplemental material). This was not observed in the other conditions, suggesting that up to three-cell ablation, compensatory mechanisms occur. On four-cell ablation, onehalf of the ablated wings shows gaps or complete migration block by $32 \mathrm{~h}$ APF (Fig. 2I; data not shown), several hours after all controls show connection (compare with Fig. $2 A-D$ ). The other half reaches the final destination but at much later time than in controls (Fig. 1C,D). Thus, four-cell ablation induces migration arrest or severe delay.

A number of issues were considered to interpret the phenotypes. First, at the time of ablation, the first four cells are very closely located to each other (Fig. $2 F$ ), thus the strong delay observed on three- or four-cell ablation cannot result from remaining cells having to cover greater distance compared with when one or two cells are ablated. Second, the ablation method does not induce unspecific effects because of nerve damaging. We submitted the axons in front of L1glia to irradiation, using the same conditions as those used to target four glia (see Materials and Methods). In none of the samples $(n=6)$ does nerve targeting affect chain migration and the MI $(25 \mathrm{~h}$ $10 \mathrm{~m} \mathrm{APF}$ ) is comparable to that of controls (Fig. 4A-D, $I-L$; Movie 2, available at www.jneurosci.org as supplemental material). For these experiments, we constructed a transgenic line that labels neurons in red (elav-DsRed express) and crossed it with repo::ncGFP animals (Fig. $4 J-L$ ), to simultaneously visualize axons and glia. Third, delay in migration does not reflect the time that it takes to get rid of cellular debris. On irradiation, targeted cells rapidly change morphology, loose contact with adjacent cells and leave their initial position, which speaks against death per se preventing invasion from adjacent cells (Aigouy et al., 2004; Soustelle et al., 2008). Finally, we performed cell ablations at several positions 
within the chain and found that they have no effect on migration, further excluding steric hindrance or toxic effects of cellular material remaining on the axons. Two- to four-cell ablation within the chain does not affect the movement of cells at the front $(n=5, \mathrm{MI}=25 \mathrm{~h}$ 19m APF) (Fig. 4; Movie 2, available at www.jneurosci.org as supplemental material). Moreover, cells on the two sides of the lesion rapidly reestablish contact by sending exploratory filopodia (Fig. $5)$. Thus, glia located posterior to the lesion move proximally and rapidly covers the gap. The fact that cells within a chain can be replaced during migration clearly demonstrates the specificity of the defects induced by tip cell ablation.

These data show that first four tip cells are major players in chain migration.

\section{Spatio-temporal features of the pioneer potential}

The observation that few wings do display connection, albeit very severely delayed, on four-cell ablation (Fig. 1C,D) raised the possibility that more than four pioneers exist and prompted us to determine their precise number.

Five tip cell-ablation indeed produces extremely severe migratory defects. Control experiments targeting the nerve in similar conditions (five impact points), however, also show migratory defects in some samples (data not shown). Thus, precise pioneer number cannot be easily established by targeting an increasing number of cells, neither can it be assessed by ablating cells behind first four, because, at $17 \mathrm{~h} \mathrm{APF}$, the chain tip changes shape and arrangement very rapidly (movie 1), preventing us from targeting cells at specific positions. We therefore asked whether cells behind first four act as pioneers at a stage at which the chain tip displays a simpler organization.

By $21 \mathrm{~h}$ APF, relative positions within the chain can be more easily assigned, the two proximal-most cells being defined as cells $+1 /+2$. Because by this stage glia have already started to migrate, we first verified that migration still requires pioneers, because this cell population may be required for triggering migration initiation but not later on. On four-cell ablation at $21 \mathrm{~h} \mathrm{APF,} 0 \%$ wings show glia connection by $28 \mathrm{~h} 30 \mathrm{~m} \mathrm{APF}(n=6)$. In two cases, we could follow migration until $30 \mathrm{~h}$ APF and still found a gap (Fig. $6 A-F$; Movie 3, available at www.jneurosci.org as supplemental material). Thus, pioneers are required throughout migration. We therefore targeted the $+6 /+7$ cell couple or the $+5 /+6$ couple and found a minor impact on migration, if any (two wings show L1-radius glial connection by $26 \mathrm{~h} \mathrm{APF}$ and one by $27 \mathrm{~h} \mathrm{APF}$ ) (Fig. $6 \mathrm{~J}, \mathrm{~K}$; Movie 3, available at www.jneurosci.org as supplemental material; and data not shown). We then targeted the $+3 /+4$ couple and confirmed their pioneer role $(n=4)$ : no wing shows connection by the time all controls do show it (Fig. $6 L-P$; Movie 3 , available at www.jneurosci.org as supplemental material).

Interestingly, although $+3 /+4$ ablated wings show gaps, radius and L1-glia are quite close, indicating that removing two pioneers is less effective than removing four, in line with results from early ablations. We then reasoned that, if the $+5 /+6$ couple were to have a pioneer role, ablating four cells, from +3 to +6 , would have a stronger impact compared with that induced by $+3 /+4$ targeting. We succeeded in two +3 to +6 ablations and found that migration is strongly delayed in one case and completely blocked in the other (data not shown). The fact that +3 to +6 ablation seems to have a stronger impact on migration than $+3 /+4$ ablation confirms that, whereas first four cells are major players, more distal ones may have a discrete pioneer role.

Late ablations reveal another unexpected feature of chain migration. On four pioneers targeting, two to four next cells display altered morphology and marked decrease in GFP labeling (Fig. $6 C-F$ ). These changes are transient, because cells slowly recover both GFP expression and morphology as they migrate toward the radius. Such phenotypes were not observed on early pioneer ablation (compare Fig. 6 with Fig. 2) (compare Movie 3 with Movie 1, available at www.jneurosci. org as supplemental material), suggesting retrograde signaling from pioneers at late stages.

In summary, five to six tip cells are required throughout migration to promote movement of follower cells.

\section{The distal cytoplasmic extension}

The presence of pioneers at the chain tip prompted us to characterize these cells further. In the absence of specific drivers and markers, we performed MARCM analyses (Lee and Luo, 2001). Conditions were set up to induce single cell, GFP-positive, clones 

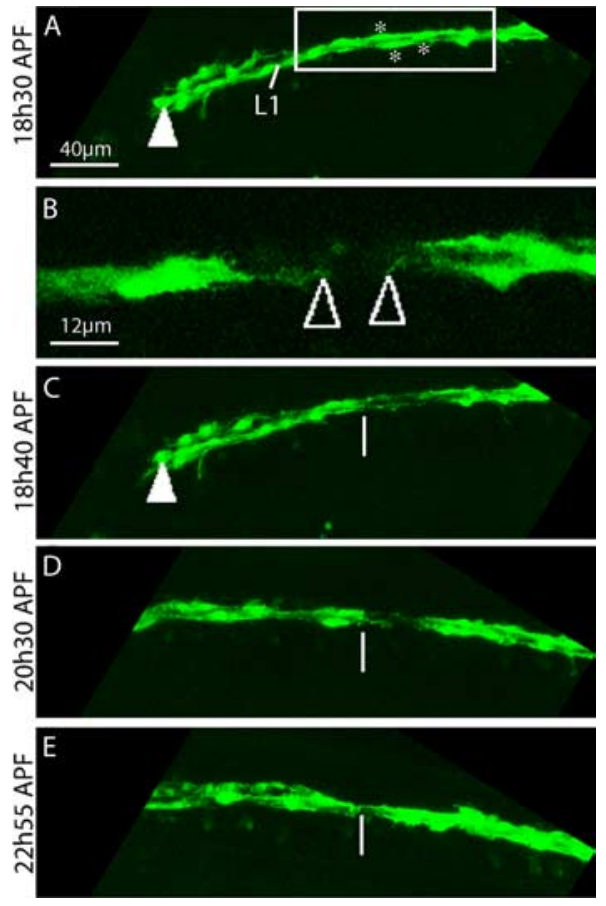

Figure 5. Ablation within the chain. An example of a three-cell ablation within the wing chain. $\boldsymbol{A}$, Cells were targeted (asterisks) at $18 \mathrm{~h} 30 \mathrm{APF}$, the white arrowhead indicates the proximal-most cell in the field. Note that this cell has left the field by 20 h30 APF. $B$ shows a high magnification of the area defined by the white rectangle in $A$ just after UV laser targeting; empty arrowheads indicate the cytoplasmic processes of the cells on each side of the lesion. $\boldsymbol{C}-\boldsymbol{E}$, The vertical bar is proximal to the lesion. Glial cells distal to the lesion fill the gap, thereby forming again a uniform chain.

and identify individual glia. Of $200 \mathrm{L1}$-glia labeled by MARCM, we retrieved eight pioneers (Fig. 7 A, C-F; supplemental Figs. 1, 2, available at www.jneurosci.org as supplemental material; and data not shown). Pioneer carries a distal cytoplasmic extension (DCE) $(n=7)$ that can be $>60 \mu \mathrm{m}$ long and contacts several follower glia. We were able to perform a time-lapse on a GFPpositive cell located at pioneer position, allowing us to follow DCE dynamics. Interestingly, whereas proximal filopodia elongate on growth and retraction, DCE remains anchored at the initial position of pioneer cell (Fig. 7C-F; Movie 4, available at www.jneurosci.org as supplemental material). Its length increases during pioneer soma translocation toward proximal positions. The finding that one MARCM clone labels a cell that displays a DCE and a cell that does not (Fig. 7C-F) may be due to the fact that only one of the two cells is a pioneer. In the future, developing conditional labeling techniques will help us targeting pioneer cells.

GFP-positive cells that are not located at the chain tip do not display a DCE $(n=192)$ (supplemental Figs. 1, 2, available at www.jneurosci.org as supplemental material) (Aigouy et al., 2004; data not shown), indicating that rear extension is not a promiscuous feature within the chain. To further confirm that the DCE is specific to pioneers we also excluded the possibility that the length of the extension is linked to the amount of migration. To this purpose, we analyzed the relationship between extension length and amount of migration by quantifying the distance over which the cell travels (the net nuclear displacement of the cell) and the cell size. In the case of the pioneer glia presenting a DCE (movie 4), the net nuclear displacement is $63 \mu \mathrm{m}$ toward the proximal side of the wing and the increase in size of the cell is of $56 \mu \mathrm{m}$ (see the last frame of movie 4), strongly indicating that there is more or less a 1:1 relationship between the traveled distance and the length of the DCE. The same analysis on two follower cells (movie 5) led to very different results. The first follower, for example, migrates over a distance of $43 \mu \mathrm{m}$ and its size has not increased at the end of the movie. Indeed, by this stage, the cell is even smaller (11 $\mu \mathrm{m}$ less long) than what it was at the beginning (see last frame of movie 5). In the case of the second follower, the cell migrates over a distance of $27 \mu \mathrm{m}$ and shows a $12 \mu \mathrm{m}$ increase in size. Interestingly, both followers keep their spindle-like shape throughout the movie and do not display a DCE.

In summary, DCE is a novel feature that characterizes pioneer cells of a migratory chain.

\section{Discussion}

Migration is a hallmark of glia of central and peripheral nervous systems. Glial chain migration entails cells moving in response to directional signals while keeping their relative position within the chain. In the present study, we use confocal time-lapse and cell ablation in the whole animal to define the cellular cues required for chain migration. Our in vivo data show for the first time that pioneer glial cells display unique features and are necessary for chain migration. This work open novel perspectives for understanding the bases of glial migration during development, as well as in pathological conditions such us regeneration, cancer and demyelinating diseases.

\section{Pioneer cells and collective chain migration}

Collective glial migration is characterized by coordinated behaviors and highly motile cells at the migratory front (Sepp et al., 2000; Silies et al., 2007). By studying the Drosophila L1 wing glia, we show that cells at the chain tip explore the environment and pave the way for the rest of the cells. The linear progression of migratory delay observed in the one- to fourcell ablations indicates that the chain requires several pioneers (Fig. 3). The presence of a pioneer population likely provides the forces that allow movement of a large group of cells stretching over considerable distance. For comparison, border cells in the fly ovary, a well studied example of collective migration (for reviews, see Montell, 2003; Rørth, 2003), form a compact unit of six to 10 equivalent cells that are in contact one with the other and a single cell extends a long process at its leading edge. Thus, border cell cluster and L1 glial chain use different strategies, likely depending on the size of the cell community. Migratory strategies also depend on the shape of the moving front. The sheet of migrating glia in the Drosophila visual system is regulated by two wrapping glia (carpet cells) that prevent glial overmigration into the eye disc (Silies et al., 2007). Interestingly, although L1 pioneers trigger migration whereas carpet cells prevent it, these examples show that glial migration requires glia-glia interactions.

The first four tip cells are crucial, few, adjacent cells, displaying a minor pioneer role. The fact that the pioneer potential decreases as distance from the tip increases suggests that pioneers respond to directional signal(s) forming a gradient or that cells at the very tip express higher levels of receptor(s) compared with more distal pioneers. Interestingly, competence to trigger migration is restricted spatially but not temporally, because pioneers are continuously required during migration. Sustained need may reflect the presence of long lasting signal(s) in the environment or continued receptor(s) activation, as has been suggested for the zebrafish lateral line, a cohesive mass of more than one hundred migrating cells 
(Haas and Gilmour, 2006). The lateral line primordium travels over a stripe of cells that express the chemokine stromal-derived factor 1 (SDF1) (Haas and Gilmour, 2006). Specific chemokine receptors expressed in leading or in trailing cells respond to SDF1 and allow lateral line migration (Valentin et al., 2007). Because such receptors have not been identified in the Drosophila genome, it will be interesting to identify the molecular pathway that is at work in the fly wing. Future studies will involve genetic screens: it will be important to determine whether growth factor pathways, which affect migration in other cell types (Montell, 2003; Rørth, 2003) or in vertebrate glia (Lai, 2005), are required in wing glia.

\section{Permissive/instructive role of pioneer cells}

Although it is clear that pioneer ablation severely delays migration, chain arrest is not a fully penetrant phenotype. Cases of connected glia were indeed found in all conditions, a phenotype that may have several explanations. Pioneer glia may play a permissive rather than an instructive role, similar to what observed for many pioneer axons. For example, elimination of a brain tract in the zebrafish embryo increases pathfinding errors by follower axons but does not prevent them from finding the correct pathway (Chitnis and Kuwada, 1991). Similarly, ablation of aCC axon in Drosophila mostly delays the formation of follower tracts (Lin et al., 1995). These pioneer axons are not absolutely required but facilitate the development of late axonal pathways. Moreover, further studies will determine whether on first four-cell ablation, adjacent cells, which normally play a minor guiding role, take over and partially compensate for the lack of pioneers. Finally, fully penetrant arrest may only be induced by very early ablations. L1-glia are born several hours before they start migrating (Aigouy et al., 2004), at a stage that is not easily accessible to UV irradiation. It is possible that pioneers provide migratory cues as soon as they differentiate. Regardless of whether pioneer glia are instructive or permissive, our analysis identifies a cell population with specific properties. In the future, the identification of mutations affecting pioneer development will shed light onto the precise nature of these cells.

\section{The distal cytoplasmic extension}

Exploratory behaviors and directional migration are provided by structures located at the leading edge, however chain migration also involves guiding neighbors. Interestingly, pioneer cells display a dynamic network of filopodia at the front of their soma (Aigouy et al., 2004) as well as a long and stable process at their rear, the DCE, in direct contact with many follower cells (present study). The identification of the DCE has several implications: first, pioneer migration occurs by soma translocation, that is, the rear end does not need to detach from its substrate. Second, DCE may provide a physical substrate and/or send a directional signal to follower cells. Third, DCE-mediated retrograde interactions may insure chain integrity, as suggested by the modification of GFP expression in follower cells on late pioneer ablation. Developing tools aiming at destabilizing DCE will help understanding its precise role and nature.

MARCM analyses strongly suggest that the DCE characterizes most pioneer cells, because only one MARCM labeled pioneer does not display this feature (supplemental Fig. $1 \mathrm{~A}$, available at www.jneurosci.org as supplemental material). DCE containing pioneers may be a widespread feature of collective migration. It will be interesting to determine whether other migrating communities display a similar structure and mode of migration.

In conclusion, our in vivo data identify the cellular cues triggering glial chain migration in Drosophila. Because glial chain migration represents an evolutionary conserved process that takes place during nervous system development and in pathological conditions (Tsai and Miller, 2002), this study sets the bases 


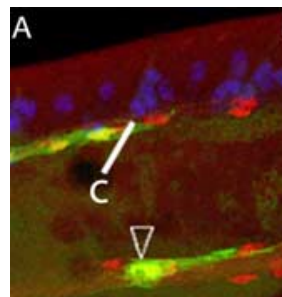

MARCM repo::ncGFP
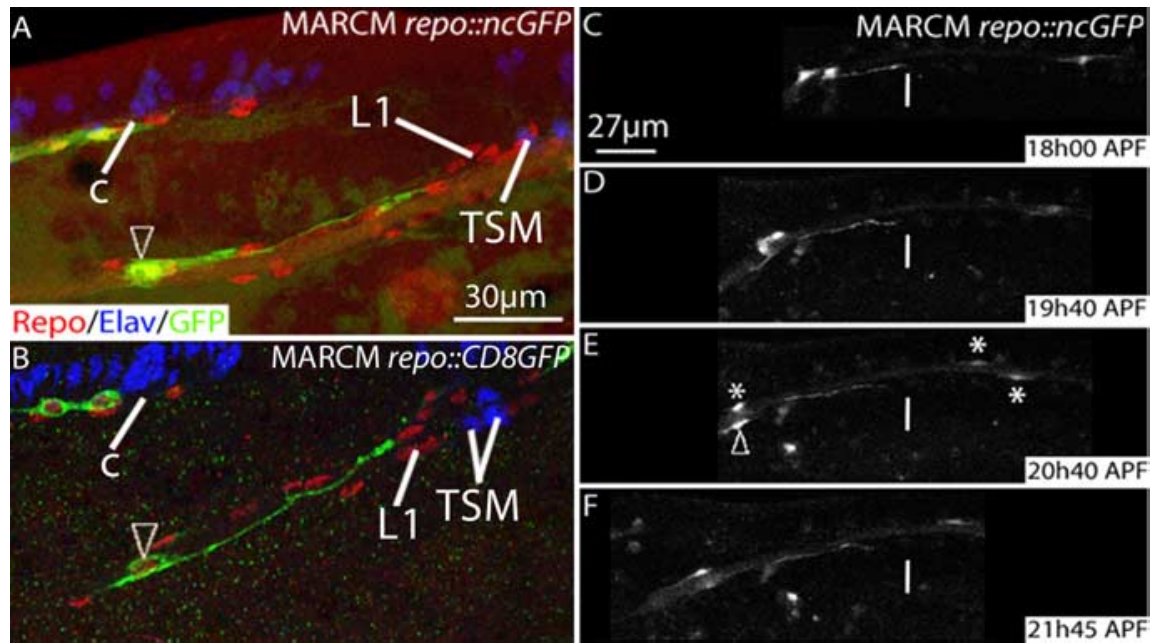

Figure 7. The distal cellular extension. MARCM identification of L1-glia in repo::ncGFP/repo::CD8GFPflies. Symbols as above. $\boldsymbol{A}$, $\boldsymbol{B}, 22 \mathrm{~h}$ APF wings labeled with anti-Repo (red: glial nuclei), anti-Elav (blue: neuronal nuclei), and anti-GFP (green: clonal glial marker). Note that pioneer cells, arrowheads in $\boldsymbol{A}, \boldsymbol{B}$, display a DCE, whereas more distal cell does not (see supplemental Fig. 2, available at www.jneurosci.org as supplemental material). C, Confocal projections from time-lapse on four GFP-positive cells, one of them displays DCE (arrowhead), the three others do not (asterisks). The vertical bar indicates the position of DCE distal tip, showing that DCE length increases on pioneer soma translocation. Scale bars: (in $\boldsymbol{A}) \boldsymbol{A}, \boldsymbol{B}, 30 \mu \mathrm{m}$; (in $\boldsymbol{C}$ ) $\boldsymbol{C}-\boldsymbol{F}, 27 \mu \mathrm{m}$.

for exploring the cellular mechanisms that drive glial chain migration in vertebrates.

\section{References}

Aigouy B, Van de Bor V, Boeglin M, Giangrande A (2004) Time-lapse and cell ablation reveal the role of cell interactions in fly glia migration and proliferation. Development 131:5127-5138.

Bevis BJ, Glick BS (2002) Rapidly maturing variants of the Discosoma red fluorescent protein (DsRed). Nat Biotechnol 20:83-87.

Chitnis AB, Kuwada JY (1991) Elimination of a brain tract increases errors in pathfinding by follower growth cones in the zebrafish embryo. Neuron $7: 277-285$.

Giangrande A (1994) Glia in the fly wing are clonally related to epithelial cells and use the nerve as a pathway for migration. Development 120:523-534.

Giangrande A, Murray MA, Palka J (1993) Development and organization of glial cells in the peripheral nervous system of Drosophila melanogaster. Development 117:895-904.

Gilmour DT, Maischein HM, Nüsslein-Volhard C (2002) Migration and
MARCM repo::ncGFP

112:595-598.

function of a glial subtype in the vertebrate peripheral nervous system. Neuron 34:577-588.

Haas P, Gilmour D (2006) Chemokine signaling mediates self-organizing tissue migration in the zebrafish lateral line. Dev Cell 10:673-680.

Lai C (2005) Peripheral glia: Schwann cells in motion. Curr Biol 15:R332-334.

Lecaudey V, Gilmour D (2006) Organizing moving groups during morphogenesis. Curr Opin Cell Biol 18:102-107.

Lee T, Luo L (2001) Mosaic analysis with a repressible cell marker (MARCM) for Drosophila neural development. Trends Neurosci 24:251-254

Lee T, Winter C, Marticke SS, Lee A, Luo L (2000) Essential roles of Drosophila RhoA in the regulation of neuroblast proliferation and dendritic but not axonal morphogenesis. Neuron $25: 307-316$

Lemke G (2001) Glial control of neuronal development. Annu Rev Neurosci 24:87-105.

Lin DM, Auld VJ, Goodman CS (1995) Targeted neuronal cell ablation in the Drosophila embryo: pathfinding by follower growth cones in the absence of pioneers. Neuron 14:707-715.

Montell DJ (2003) Border-cell migration: the race is on. Nat Rev Mol Cell Biol 4:13-24.

Rørth P (2003) Communication by touch: role of cellular extensions in complex animals. Cell

Sepp KJ, Schulte J, Auld VJ (2000) Developmental dynamics of peripheral glia in Drosophila melanogaster. Glia 30:122-133.

Silies M, Yuva Y, Engelen D, Aho A, Stork T, Klämbt C (2007) Glial cell migration in the eye disc. J Neurosci 27:13130-13139.

Soustelle L, Aigouy B, Asensio ML, Giangrande A (2008) A UV laser mediated cell selective destruction by confocal microscopy. Neural Dev 3:11.

Tsai HH, Miller RH (2002) Glial cell migration directed by axon guidance cues. Trends Neurosci 25:173-175, discussion 175-176.

Valentin G, Haas P, Gilmour D (2007) The chemokine SDF1a coordinates tissue migration through the spatially restricted activation of Cxcr7 and Cxcr4b. Curr Biol 17:1026-1031.

Van De Bor V, Walther R, Giangrande A (2000) Some fly sensory organs are gliogenic and require glide $/ \mathrm{gcm}$ in a precursor that divides symmetrically and produces glial cells. Development 127:3735-3743.

von Hilchen CM, Beckervordersandforth RM, Rickert C, Technau GM, Altenhein B (2008) Identity, origin, and migration of peripheral glial cells in the Drosophila embryo. Mech Dev 125:337-352. 